\title{
Modelagem numérica do comportamento de ligações com parafusos auto-atarraxantes em X em corpos de prova de MLC com madeiras do tipo Eucalipto urograndis
}

\author{
Numerical modeling of connections behavior \\ with self-tapping screws disposed in $\mathrm{X}$ \\ position in specimens of MLC with \\ Eucalyptus urograndis woods
}

Carlito Calil Neto ${ }^{1}$, Julio Cesar Molina ${ }^{2}$,

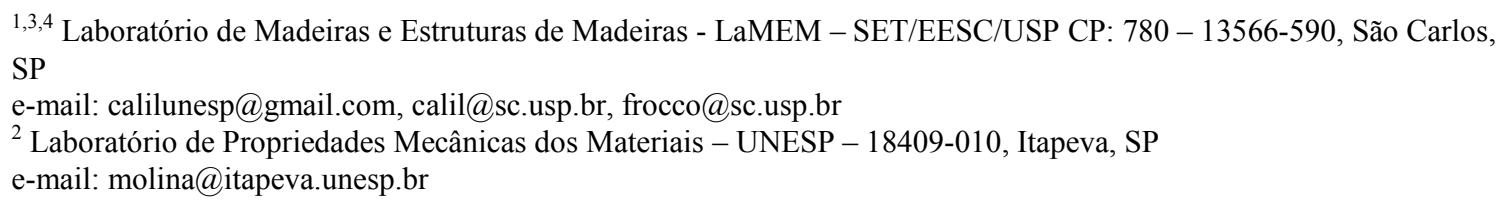

\begin{abstract}
RESUMO
Neste trabalho avaliou-se numericamente, a partir da utilização do software ANSYS, versão 10.0, o comportamento de ligações metálicas efetuadas por parafusos auto-atarraxantes com diâmetro igual a $11 \mathrm{~mm}$, dispostos em X, em corpos de prova confeccionados com madeira de reflorestamento do tipo Eucalipto urograndis. A rigidez $\mathrm{k}$ (força versus deslizamento) do sistema de ligação foi avaliada, neste caso, por meio da simulação de ensaios de cisalhamento direto (direct shear), considerando-se $1 / 2$ estrutura com carregamento aplicado até a ruptura do corpo de prova. A estratégia de se utilizar $1 / 2$ estrutura simétrica teve o objetivo de reduzir o tempo de processamento numérico do modelo de corpo de prova. Na modelagem da madeira e dos conectores de aço foi utilizado o elemento finito solid45. Os resultados da análise numérica foram comparados com resultados obtidos a partir de ensaios experimentais realizados em corpos de prova de madeira com a mesma configuração e carregamentos utilizados na análise experimental. A calibração do modelo numérico foi realizada a partir da comparação da curva numérico-experimental "força aplicada" versus "deslizamento obtido". Os resultados numéricos obtidos para a resistência e rigidez k do sistema de ligação X mostraram uma boa concordância com os resultados experimentais, sendo que os resultados experimentais foram relativamente mais conservadores que os resultados numéricos. Como resultado apresenta-se, principalmente, a estratégia de modelagem numérica utilizada, neste caso.
\end{abstract}

Palavras-chave: Conectores de cisalhamento, parafusos auto-atarraxantes, análise numérico-experimental, madeira de reflorestamento.

\footnotetext{
ABSTRACT

In this work was evaluated by numerical simulation, from the use of ANSYS software, version 10.0, the behavior of steel connections made by self-tapping screws with diameter of and $11 \mathrm{~mm}$, in X position, in specimens made with reforested wood of the type Eucalyptus urograndis. The stiffness $\mathrm{k}$ (force versus displacement) of the connection system was evaluated, in this case, by direct shear testing simulation (direct shear), considering $1 / 2$ structure with applied load until the rupture of the specimen. The strategy of using $1 / 2$ symmetrical structure of specimen aimed to reduce the time of numeric processing of the specimen. In the modeling of wood and steel connectors was used the finite element solid45. The numerical analysis results were compared with results obtained from experimental tests on wood specimens with same configuration and loadings used in experimental analysis. The calibration of the numerical model was made from the comparison of numerical and experimental curves "applied force" versus "obtained displacement". The numerical results for the strength and stiffness $\mathrm{k}$ of the connection system analyzed X showed a good agreement with the experi-
} 
mental results and the values of experimental results were fairly smaller than the numerical values. As a result presents mainly the entire numerical modeling strategy used in this case.

Keywords: Shear connections, self-tapping screws, numeric analysis-experimental, reforestation wood.

\section{INTRODUÇÃO}

Segundo MOLINA [1], o estudo das ligações para as estruturas de madeira tem, no Brasil, grande interesse nos meios técnico e cientifico, pois, é na região das ligações que ocorrem os maiores problemas nas estruturas de madeira. A utilização de vários tipos de conectores para estruturas de madeira faz com que haja cada vez mais interesse na avaliação do comportamento do sistema estrutural considerado. Assim vários tipos de abordagens podem ser considerados na análise das ligações para estruturas de madeira, seja no âmbito experimental, analítico como também numérico. Atualmente, com o avanço dos microcomputadores e dos softwares, estudos de caráter numérico têm ocorrido com maior frequência. Dentre as ferramentas existentes para análise numérica destacam-se programas comerciais ou softwares específicos (SAP2000, ANSYS, ABAQUS, etc.) desenvolvidos com base no Método dos Elementos Finitos. Esses softwares possibilitam reproduzir numericamente o comportamento das estruturas, evitando-se custos inerentes à realização de ensaios experimentais sem a necessidade do desenvolvimento analítico, em geral, descritos por equacionamentos custosos, em razão da considerável complexidade da análise nos campos das tensões e das deformações.

Ainda, de acordo com MOLINA [1], por outro lado, a maioria dos modelos numéricos propõe formas simplificadas de análise sem considerar os critérios de resistência dos materiais. Nesse sentido, os modelos procuram, de uma maneira geral, restringir o comportamento da estrutura a limites linearmente elásticos, ao invés de considerar os verdadeiros critérios de falha dos materiais obtendo, dessa forma, respostas muito conservadoras. Pode-se dizer ainda, que, as estruturas assim analisadas, são capazes de resistir a esforços, significativamente, superiores aos esforços de projeto. Porém, as tensões e deformações geradas pelas solicitações externas, frequentemente, excedem os limites elásticos dos materiais e, portanto, a resposta estrutural dos materiais não deveria ser estudada como linearmente elástica. Assim, os resultados obtidos por uma análise linear são válidos, à medida que as deformações plásticas são pequenas. Somado a esses fatores, a geometria dos elementos estruturais, especialmente nas regiões próximas as conexões, é capaz de elevar os estados de tensões a níveis máximos, atingindo a falha dos materiais para carregamentos de serviço. Por esta razão, na modelagem numérica de estruturas, é necessária a utilização de modelos sofisticados que permitam determinar, com maior precisão, o verdadeiro comportamento estrutural dos materiais, principalmente nas regiões das conexões. Portanto, para carregamentos de serviço, os modelos devem considerar o comportamento nãolinear nos trechos inelásticos, a plastificação, além dos efeitos de ortotropia dos materiais, quando necessários, procurando, deste modo, evitar possíveis deficiências em condições para projeto. De uma maneira geral, a análise numérica tem avançado bastante no sentido de descrever os fenômenos reais das estruturas.

De acordo com FRANGI et al. [2], na composição de conexões para estruturas de madeira podem ser utilizados pregos, parafusos, barras de aço, entre outros. Os conectores também podem ser usados para estruturas mistas de madeira e concreto. Segundo THOMAS e HANS [3] um dos tipos de conexão é aquela formada por parafusos auto-atarraxantes dispostos na posição vertical ou inclinados em relação às fibras da madeira. Quando posicionados na posição vertical, os esforços de cisalhamento na interface da madeira provocam embutimento na madeira e/ou flexão no conector metálico, na direção do fluxo de cisalhamento. Segundo MOLINA [1], nos parafusos inclinados, os mecanismos de deformações das conexões tracionadas também provocam embutimento na madeira, enquanto que os parafusos comprimidos formam rótulas plásticas, limitando a sua resistência, além do embutimento na madeira. Portanto, para o conector inclinado, a solicitação axial de tração é a que melhor se aproxima das condições de serviço do conector. Para os conectores verticais é interessante utilizar barras com maiores diâmetros visando diminuir o efeito de plastificação do pino metálico.

O principal objetivo deste trabalho foi o desenvolvimento de um modelo numérico, tridimensional, construído a partir do software ANSYS [4], para a verificação do comportamento do sistema de ligação em X, efetuado por parafusos auto-atarraxantes com diâmetro de $11 \mathrm{~mm}$, em corpos de prova de madeiras de eucalipto urograndis. Como resultados, são apresentados os níveis de tensões nas regiões da ligação metálica, como também a rigidez (Força/deslizamento) do sistema comparada à resposta fornecida por ensaios experimentais. Espera-se que este trabalho possa auxiliar, principalmente, na avaliação das distribuições das tensões nas regiões dos conectores de cisalhamento, as quais não são observadas facilmente, somente a partir de ensaios experimentais. 


\section{MATERIAIS E MÉTODOS}

\subsection{Programa experimental}

Para comparação com os resultados numéricos de resistência e rigidez das ligações, foram confeccionados corpos de prova com madeira de Eucalipto urograndis (feitos em Madeira Laminada Colada, utilizando-se, na colagem das lâminas, resina Fenol Resorcinol, tendo a madeira utilizada, neste caso, densidade de $637 \mathrm{~kg} / \mathrm{m}^{3}$, módulo de elasticidade $1900 \mathrm{kN} / \mathrm{cm}^{2}$ e resistência a compressão de 7,78 kN/ $\mathrm{cm}^{2}$ ) com conexões em X (dois pares de conectores) efetuadas por parafusos auto-atarraxantes com diâmetro de $11 \mathrm{~mm}$ e comprimento de 200m, conforme apresentado na Figura 3. A inclinação de cada um dos parafusos utilizados na composição dos conectores $\mathrm{X}$ foi de $45^{\circ}$, conforme ilustra a configuração do corpo de prova apresentada na Figura 1.

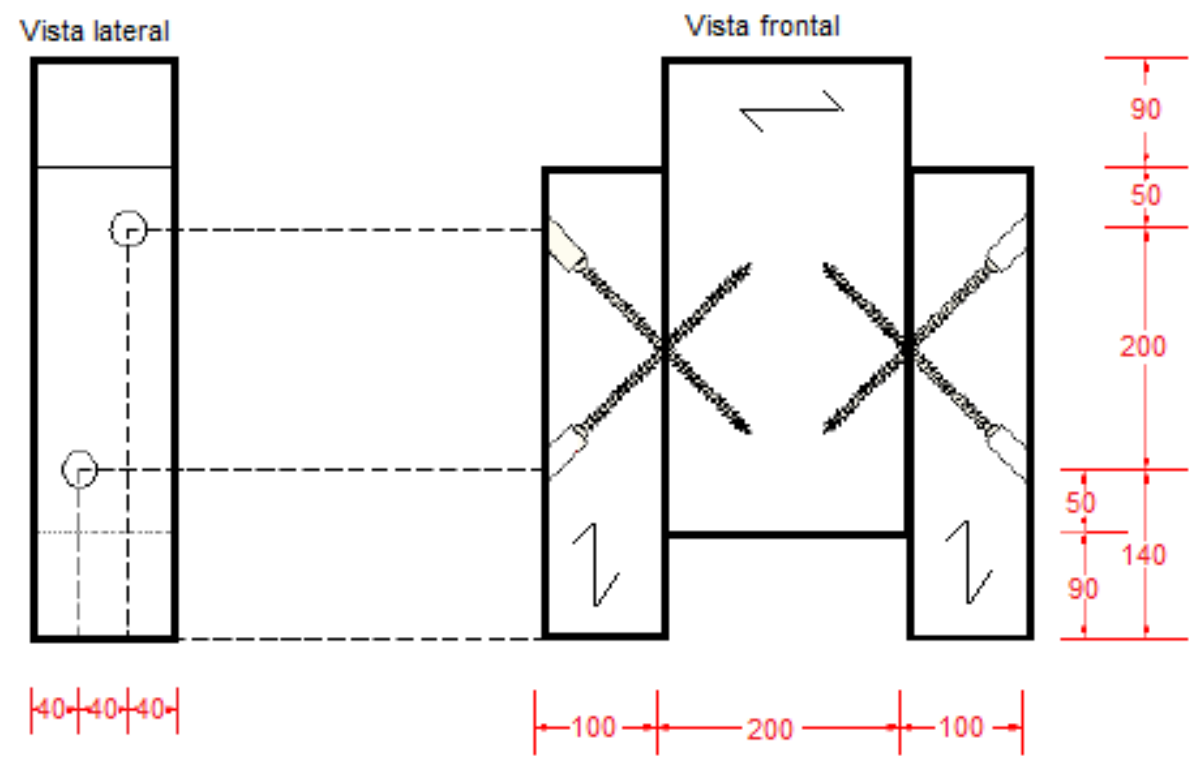

Figura 1: Configuração do corpo de prova com parafusos auto-atarraxantes em $\mathrm{X}$, peça central de madeira com fibras horizontais e peças laterais com fibras na direção vertical. Fonte: CALIL NETO [5].

Na Figura 2 apresenta-se o modelo de corpo de prova utilizado com sistema de conexão metálico formado por dois pares em X (Parafusos auto-atarraxantes da marca Rothoblass) tendo as peças centrais de MLC com fibras horizontais, ou seja, posicionadas a $90^{\circ}$ com relação às fibras das peças laterais de MLC. 


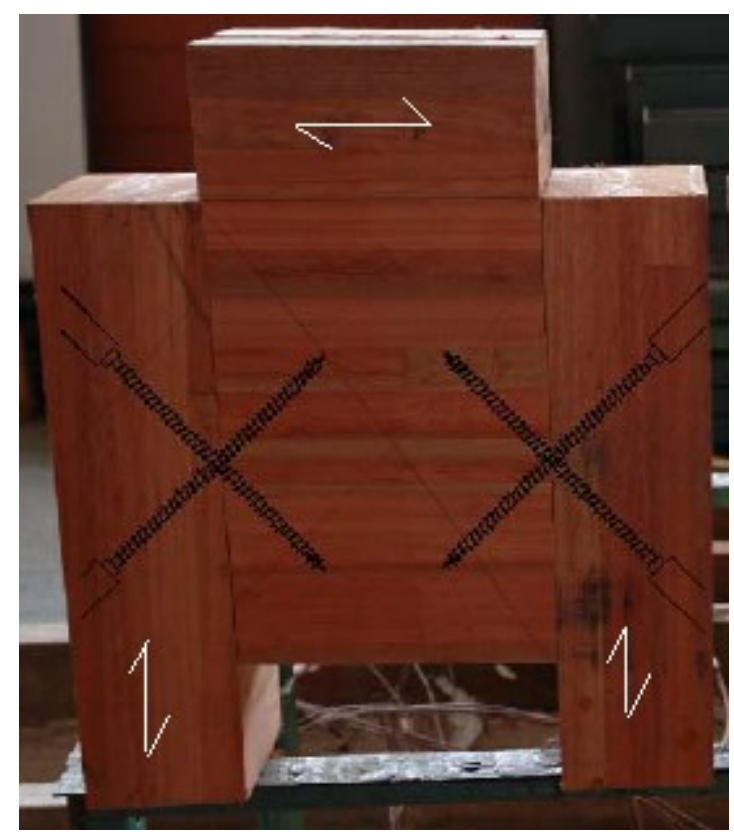

Figura 2: Modelo de corpo de prova utilizado nos ensaios experimentais de resistência e rigidez da ligação em X. Fonte: CALIL NETO [5].

Na Figura 3 apresentam-se os detalhes e dimensões do conector (da marca Rothoblass) utilizado na composição dos pares de conectores em X. Os parafusos, neste caso, foram fixados com auxílio de uma furadeira e gabarito de furação sem a utilização de resinas.

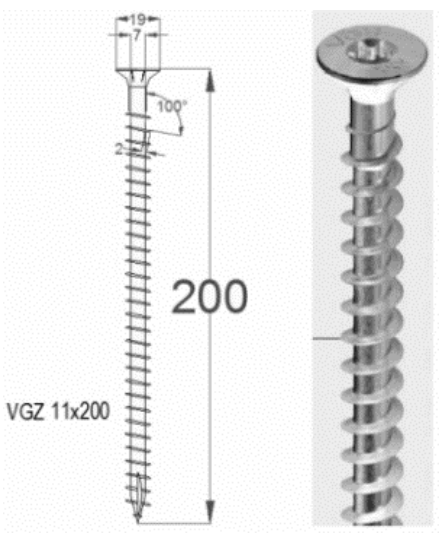

Figura 3: Parafuso Auto-atarraxante VGZ. Fonte: ROTHOBLASS [6].

Na condução dos ensaios estáticos de cisalhamento dos corpos de prova foi utilizado um pórtico de reação, com cilindro hidráulico com capacidade de $480 \mathrm{kN}$ (48.000kgf), acionado por um atuador de controle manual e sistema de aquisição de dados externo. O sistema de aquisição de dados externo utilizado, SYSTEM 5000, de 20 canais. Três deles foram utilizados para a recepção dos sinais, sendo um para a célula de carga, e os outros dois para os transdutores de deslocamentos. Na Figura 4 estão apresentados: o sistema de aquisição de dados utilizado e o esquema de instrumentação dos corpos de prova. 


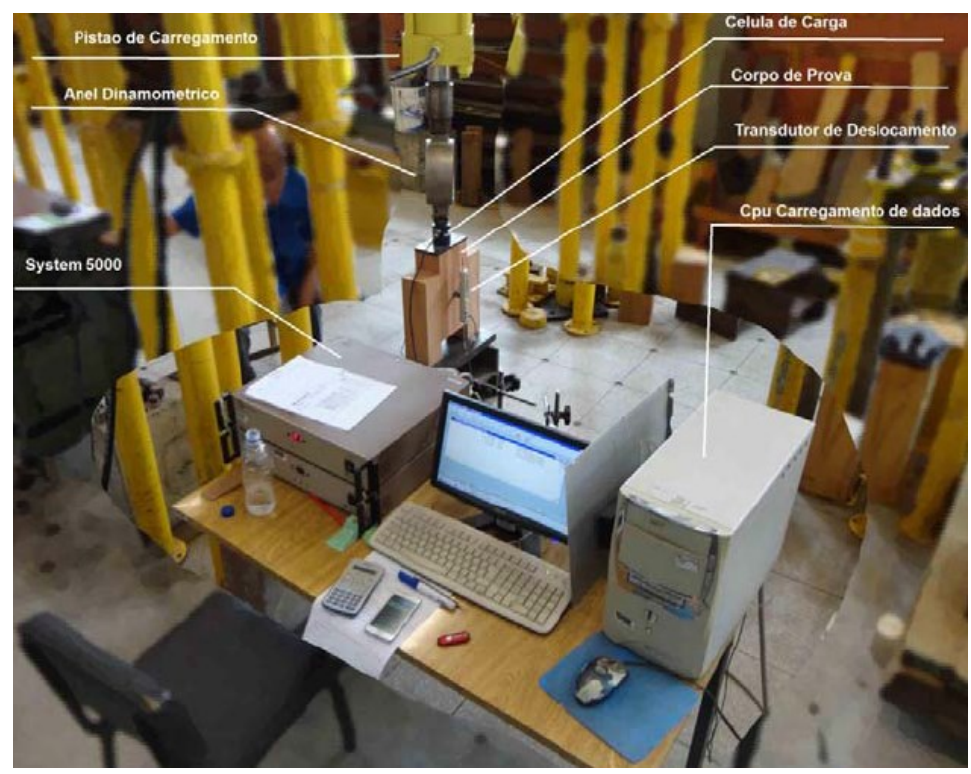

Figura 4: Sistema de aquisição de dados SYSTEM 5000. Fonte: CALIL NETO [5].

Os ensaios de cisalhamento foram realizados em três ciclos de carga, sendo os dois primeiros ciclos com carga aplicada até 50\% da resistência estática da ligação, conforme recomendações da ABNT NBR 7190 [7] (determinada inicialmente a partir de um corpo-de-prova-gêmeo), e o terceiro ciclo, com carregamento aplicado até a ruptura. A velocidade de aplicação de carga, neste caso, foi de $0,10 \mathrm{kN} / \mathrm{mm}$. A resistência da ligação foi determinada, convencionalmente, pela força aplicada ao corpo de prova padronizado, que provoca na ligação uma deformação específica residual de $2 \%$. Foram realizados três ciclos de carga e descarga, sendo os dois primeiros ciclos com carga entre $10 \%$ e $50 \%$ da força de ruptura, obtida para um corpo de prova gêmeo e, o terceiro ciclo, conduzido até a ruptura do corpo de prova. A Figura 12 mostra os resultados obtidos neste caso. O sistema de solicitação do corpo-de-prova foi desenvolvido por meio do pórtico de reação, acionado manualmente e, os carregamentos estáticos, foram controlados por meio de uma célula de carga com capacidade de $150 \mathrm{kN}$. Os deslocamentos entre as peças de madeira foram medidos por meio de dois transdutores de deslocamentos, com sensibilidade de $0,001 \mathrm{~mm}$ e curso máximo de $5 \mathrm{~mm}$, posicionados em faces opostas do corpo-de-prova, como mostra a Figura 5.

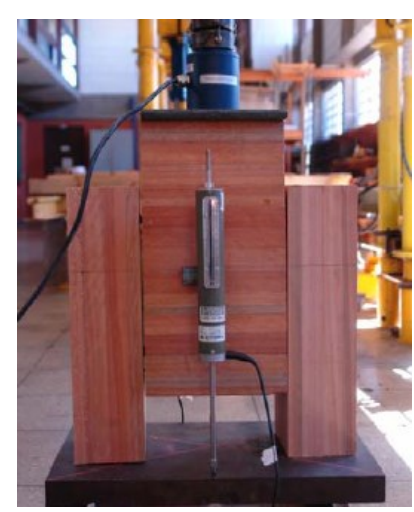

Figura 5: Instrumentação do corpo de prova com transdutores de deslocamento e célula de carga. Fonte: CALIL NETO $[5]$.

Os ensaios experimentais e numéricos foram realizados no Laboratório de Madeiras e de Estruturas de Madeira da Escola de Engenharia de São Carlos da Universidade de São Paulo Ressalta-se que os resultados apresentados nesse manuscrito fazem parte de um trabalho completo sobre ligações com parafusos autoatarraxantes, considerando duas espécies (Eucalipto urograndis e Pinus sp), diferentes inclinações para a fixação dos conectores $\left(45^{\circ}\right.$ e $\left.90^{\circ}\right)$, além de diferentes diâmetros de conectores de cisalhamento ( $9 \mathrm{~mm}$ e 11 $\mathrm{mm})$. Todas as informações e detalhes sobre esse trabalho podem ser encontradas em CALIL NETO [5]. Vale mencionar ainda que a análise experimental realizada por CALIL NETO [5] foi acompanhada de uma 
análise estatística, para investigação dos fatores individuais mais influentes na determinação dos valores de força última, obtidos para os corpos de prova de cisalhamento analisados. Os fatores experimentais considerados, neste caso, foram: Espécie de Madeira (Eucalipto urograndis e Pinus sp), orientação das fibras (horizontal e vertical), diâmetro do parafuso $(9 \mathrm{~mm}$ e $11 \mathrm{~mm})$ e inclinação do parafuso $\left(45^{\circ}\right.$ e $\left.90^{\circ}\right)$, conduzindo a um planejamento fatorial completo do tipo 24 , com 16 tratamentos (T) distintos, conforme explicitados na Tabela 1.

Tabela 1: Constantes admitidas para o aço dos conectores no modelo numérico. Fonte: CALIL NETO [5]

\begin{tabular}{|c|c|c|c|c|}
\hline TRATAMENTO & MADEIRA & $\begin{array}{l}\text { ORIENTAÇÃO DAS FIBRAS } \\
\text { (PEÇA CENTRAL) }\end{array}$ & $\begin{array}{l}\text { DIÂMETRO } \\
\text { DO PARAFUSO }\end{array}$ & $\begin{array}{l}\text { INCLINAÇÃO } \\
\text { DO PARAFUSO }\end{array}$ \\
\hline $\mathrm{T} 1$ & E. urograndis & Vertical & $9 \mathrm{~mm}$ (milímetro) & $45^{\circ}$ (grau) \\
\hline $\mathrm{T} 2$ & E. urograndis & Vertical & $9 \mathrm{~mm}$ (milímetro) & $90^{\circ}$ (grau) \\
\hline $\mathrm{T} 3$ & E. urograndis & Vertical & $11 \mathrm{~mm}$ (milímetro) & $45^{\circ}$ (grau) \\
\hline $\mathrm{T} 4$ & E. urograndis & Vertical & $11 \mathrm{~mm}$ (milímetro) & $90^{\circ}$ (grau) \\
\hline $\mathrm{T} 5$ & E. urograndis & Horizontal & $9 \mathrm{~mm}$ (milímetro) & $45^{\circ}$ (grau) \\
\hline T6 & E. urograndis & Horizontal & $9 \mathrm{~mm}$ (milímetro) & $90^{\circ}$ (grau) \\
\hline $\mathrm{T} 7$ & E. urograndis & Horizontal & $11 \mathrm{~mm}$ (milímetro) & $45^{\circ}$ (grau) \\
\hline $\mathrm{T} 8$ & E. urograndis & Horizontal & $11 \mathrm{~mm}$ (milímetro) & $90^{\circ}$ (grau) \\
\hline T9 & Pinus sp & Vertical & $9 \mathrm{~mm}$ (milímetro) & $45^{\circ}$ (grau) \\
\hline $\mathrm{T} 10$ & Pinus sp & Vertical & $9 \mathrm{~mm}$ (milímetro) & $90^{\circ}$ (grau) \\
\hline $\mathrm{T} 11$ & Pinus sp & Vertical & $11 \mathrm{~mm}$ (milímetro) & $45^{\circ}$ (grau) \\
\hline $\mathrm{T} 12$ & Pinus sp & Vertical & $11 \mathrm{~mm}$ (milímetro) & $90^{\circ}$ (grau) \\
\hline $\mathrm{T} 13$ & Pinus sp & Horizontal & $9 \mathrm{~mm}$ (milímetro) & $45^{\circ}$ (grau) \\
\hline $\mathrm{T} 14$ & Pinus sp & Horizontal & 9 mm (milímetro) & $90^{\circ}$ (grau) \\
\hline $\mathrm{T} 15$ & Pinus sp & Horizontal & $11 \mathrm{~mm}$ (milímetro) & $45^{\circ}$ (grau) \\
\hline $\mathrm{T} 16$ & Pinus sp & Horizontal & $11 \mathrm{~mm}$ (milímetro) & $90^{\circ}$ (grau) \\
\hline
\end{tabular}

Assim, para investigar a influência dos fatores individuais e das interações nos valores das forças dos ensaios das ligações, foi utilizada a análise de variância (ANOVA) considerada ao nível de significância $(\alpha)$ de 5\% tendo a equivalência entre os valores médios das forças como hipótese nula (H0) e a não equivalência (H1) como hipótese alternativa. P-valor ao nível de significância implica em rejeitar H0, aceitando-se em caso contrário. No caso de serem significativos os fatores sobre os valores das forças obtidas, foi utilizado o teste de comparações múltiplas de Tukey, de maneira a evidenciar os melhores dentre os níveis do fator. Para a validação da ANOVA foram avaliadas a normalidade (Teste de Anderson-Darling) na distribuição dos valores das forças e a homogeneidade entre variâncias dos tratamentos (testes de Bartlett e Levene).

\subsection{Programa numérico}

Na modelagem do sistema misto analisado, utilizou-se o software ANSYS [4], versão 10.0. A opção pela utilização do referido software se deu pelo fato das ferramentas disponibilizadas pelo mesmo permitirem a análise do modelo em regime de não linearidade física e também geométrica, além da utilização de elementos de contato entre os materiais. Por outro lado, a escolha de cada elemento finito utilizado foi feita a partir da consideração de sua representatividade perante o comportamento a ser simulado. Os elementos finitos utilizados, neste caso, são disponibilizados na biblioteca interna do ANSYS [4].

O modelo numérico desenvolvido neste trabalho foi constituído por dois principais grupos de elementos: peças de madeira e conectores de cisalhamento, conforme configuração utilizada na análise experimental. Um terceiro grupo de elementos foi constituído pelos elementos de contato na interface entre os materiais. Todos os elementos de madeira e aço, utilizados na configuração do modelo de corpo de prova, foram discretizados separadamente, porém, de forma a coincidir os nós de cada um na interface entre eles, possibilitando o acoplamento entre tais elementos, realizado por meio destes nós. As malhas dos materiais que compõem o modelo foram geradas e discretizadas no software TRUEGRID [8], versão 2.10, sendo, posteriormente, exportadas para o ANSYS [4], onde receberam as correspondentes propriedades dos materiais, condições de vinculação e carregamentos, necessárias para a análise dos sistemas mistos em questão. O TRUEGRID [8] é 
um software específico para a construção de malhas de elementos finitos e, este, foi uma ferramenta fundamental na geração das malhas dos elementos. Essa utilização foi devido à necessidade de obtenção de um maior grau de refinamento nas regiões das conexões, o que não foi possível de ser conseguido somente com a utilização das ferramentas do ANSYS [4]. A Figura 6a mostra os detalhes da malha utilizada para representar os corpos de prova de cisalhamento analisados com conectores dispostos em X. A Figura $6 \mathrm{~b}$ ilustra a metade do corpo de prova considerada na simulação numérica.

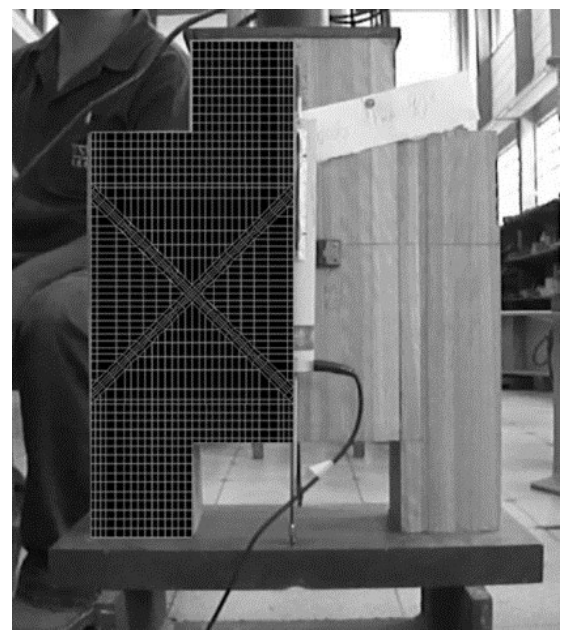

(a)

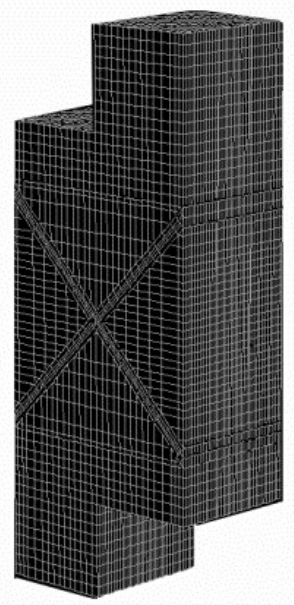

(b)

Figura 6: Criação da malha de elementos finitos no software TRUEGRID [8].

Para tanto, foram criados scripts (para ambos os softwares: TRUEGRID [8] e ANSYS [4]), contendo as sequências de comandos para a geração e análise automática dos modelos. Os comandos necessários para a análise dos modelos numéricos no ANSYS [4] foram escritos em função das propriedades elásticas e plásticas dos materiais, do comportamento das curvas experimentais tensão versus deformação, entre outros, os quais variam de um modelo para outro. A conversão da linguagem de programação do TRUEGRID [9] para o ANSYS [3] foi realizada através da utilização do software TGEDIT [9]. A malha do modelo numérico foi analisada a partir de diferentes níveis de refinamento, até que os resultados conduzissem a respostas satisfatórias em termos de deslocamentos e de deformações. Nas regiões das conexões as malhas foram mais discretizadas com relação as outras regiões do modelo. O modelo numérico em questão considerou $1 / 2$ estrutura (simétrica) devidamente vinculada, tendo em vista a diminuição do tempo de processamento. Na Figura 7 são apresentados os detalhes de $1 / 2$ estrutura do corpos-de-prova com o posicionamento dos conectores dispostos em X, com 45 graus, para o modelo numérico desenvolvido.

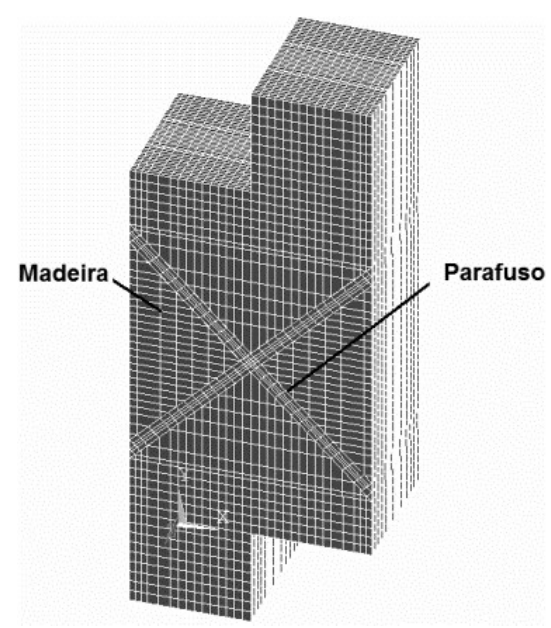

(a)

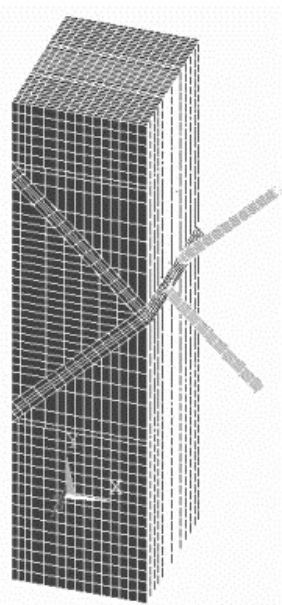

(b)

Figura 7: Representação do modelo no ANSYS [4]: (a) $1 \frac{1}{2}$ simétrica do corpo de prova; (b) parafusos auto-atarraxantes inclinados a $45^{\circ}$ no interior da madeira. 
Na discretização das peças de madeira e dos conectores de cisalhamento no ANSYS [4] utilizou-se o elemento solid45. O elemento solid45 consiste num elemento hexaédrico, com oito nós, tendo cada nó três graus de liberdade (translações segundo os eixos x, y e z), e permite, ainda, a consideração de efeitos importantes como, por exemplo, plasticidade e ortotropia para os materiais. Os elementos conta173 e targe 170 foram utilizados a fim de representar os contatos existentes com possíveis deslocamentos na interface: madeira-madeira. Esses elementos são utilizados em análises tridimensionais, com contato do tipo superfíciesuperfície, que surge do trabalho em conjunto dos elementos targe170 (definido pelo ANSYS como superfície alvo) e conta173 (definido como superfície de contato). Esses elementos são capazes de simular a existência de pressão entre os elementos, quando há contato, e separação entre os mesmos elementos, quando não há contato. O par de contatos utilizado permite, ainda, a consideração do atrito entre as partes. A Figura 8 mostra os detalhes dos elementos finitos considerados pelo ANSYS [4].

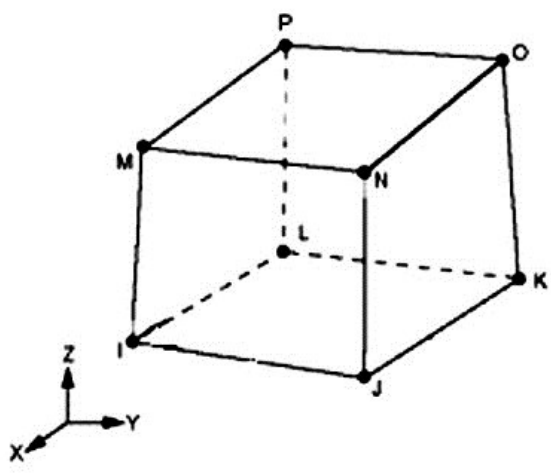

(a)

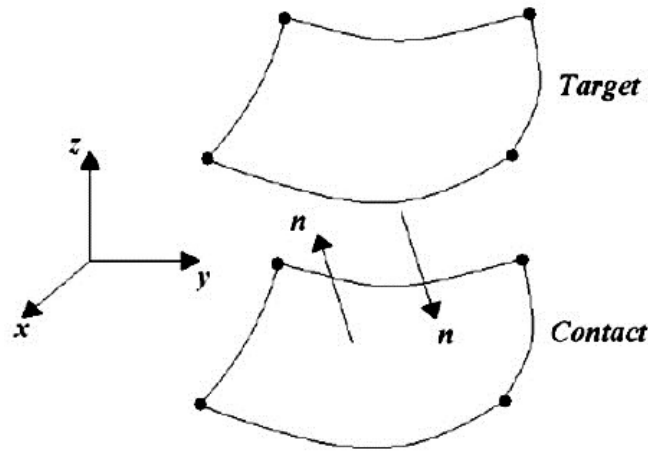

(b)

Figura 8: Elementos finitos utilizados no modelo numérico: (a) Solid45; (b) Conta173 e Targe170. Fonte ANSYS [4].

Para a madeira optou-se pela consideração de um comportamento isotrópico (mesmas propriedades físicas para cada direção considerada) para a madeira, com a utilização do critério de Von Mises. O modelo constitutivo, adotado para a madeira, simulou um comportamento elasto-plástico, através de curvas bi-lineares, dependendo das direções principais do material, conforme Figura 9.

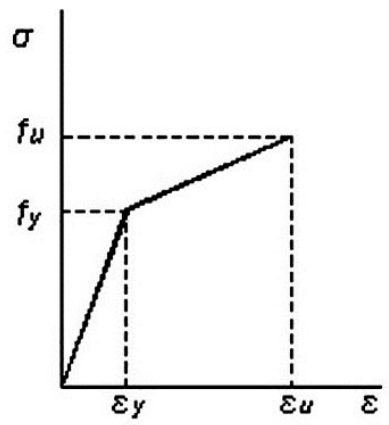

Figura 9: Modelo constitutivo adotado para a madeira. Fonte: MOLINA [1].

Admitiu-se no modelo numérico, para o valor da tensão última de plastificação da madeira $\left(\sigma_{\mathrm{y}}\right)$, na direção das fibras, o valor da resistência da madeira na compressão $\left(\sigma_{\mathrm{y}}=7,78 \mathrm{kN} / \mathrm{cm}^{2}\right)$, sendo $\mathrm{E}_{\mathrm{y}}$ o módulo de elasticidade na direção das fibras $\left(\mathrm{E}_{\mathrm{y}}=1900 \mathrm{kN} / \mathrm{cm}^{2}\right.$. No ANSYS [4], o eixo de coordenadas " $\mathrm{y}$ " correspondeu à direção longitudinal das fibras da madeira, o eixo "z" à direção radial, e o eixo "x" à direção tangencial. Assim, de acordo com BALLARIN [10] e DIAS [11], algumas relações entre as propriedades adotadas nesse trabalho para a madeira foram: $\mathrm{G}=\left(\mathrm{E}_{\mathrm{y}} / 15\right) ; v=0,23$ e $\mathrm{G}_{\mathrm{T}}=1,8$, sendo: $\mathrm{E}_{\mathrm{y}} \pm=$ módulo de elasticidade na direção longitudinal (compressão); $v=$ coeficiente de Poison; $G=$ módulo de elasticidade transversal e $G_{\mathrm{T}}=$ módulo tangente de corte. As propriedades físicas e mecânicas, necessárias para a calibração do modelo no ANSYS, foram obtidas a partir da caracterização dos materiais na análise experimental. Por simplicidade, os comportamentos da madeira, na tração e na compressão, foram considerados iguais. Para os conectores de cisalhamento foi adotado um modelo bi-linear (Figura 10), com encruamento isótropo e critério de plastifica- 
ção de von Mises. Conforme descrito em FLORES et al. [12] e MOLINA [1], a caracterização do comportamento isotrópico admitido para do aço exigiu a utilização de quatro constantes: E (Módulo de elasticidade), $\sigma_{\mathrm{p}}$ (tensão de plastificação), $\mathrm{E}_{\mathrm{T}}$ (módulo tangente) e $v$ (coeficiente de Poison) conforme Tabela 2.

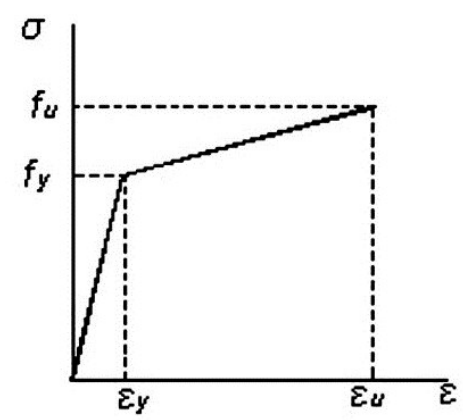

Figura 10: Modelo constitutivo adotado para o aço dos conectores de cisalhamento. Fonte: MOLINA [1].

Tabela 2: Constantes admitidas para o aço dos conectores no modelo numérico.

\begin{tabular}{|c|c|c|}
\hline PARÂMETRO & VALOR & UNIDADE \\
\hline $\mathrm{E}$ & 21000 & \multirow{4}{*}{ Kilonewton por centímetro quadrado $\left(\mathrm{kN} / \mathrm{cm}^{2}\right)$} \\
\hline$\sigma_{\mathrm{p}}$ & 50 & \\
\hline ET & 380 & \\
\hline$v$ & 0,3 & \\
\hline$\rho$ & $7,85 \mathrm{E}^{-05}$ & Kilonewton por centímetro cúbico $\left(\mathrm{kN} / \mathrm{cm}^{3}\right)$ \\
\hline
\end{tabular}

Para garantir a estabilidade do modelo durante a aplicação do carregamento foram respeitadas as condições de simetria e também de vinculação dos nós dos apoios, conforme ilustrado na Figura 11. O modelo analisado foi vinculado em concordância com os ensaios experimentais. Vale lembrar que os nós dos elementos utilizados nos modelos apresentam apenas três graus de liberdade que são referentes às translações em $\mathrm{x}$, y e z (coordenadas locais). A simulação numérica foi dividida em duas etapas. Na primeira efetuou-se a calibração do modelo numérico a partir do carregamento estático utilizado nos ensaios realizados. A validação do modelo foi efetuada a partir da comparação do comportamento das curvas Força versus Deslocamento, conforme apresentado na Figura 13.

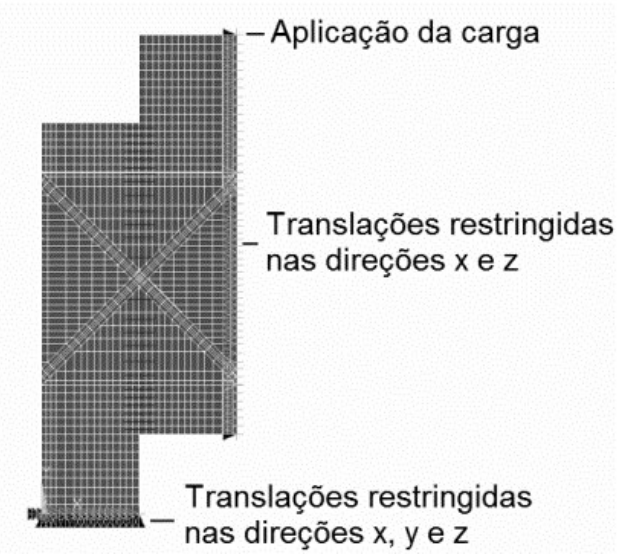

Figura 11: Condições de vinculação de $1 \frac{1}{2}$ corpo de prova com conectores dispostos em X.

O carregamento no modelo numérico foi aplicado de forma incremental, devido à consideração da não linearidade física dos materiais madeira e concreto. $\mathrm{O}$ incremento de carga foi controlado utilizando-se o recurso do ANSYS [4] denominado "Automatic Time Stepping". De acordo com a documentação do ANSYS [3], o recurso citado reduz o valor do incremento, quando a previsão do número de iterações ultrapassa o li- 
mite estabelecido, caso se obtenha incrementos de deformações plásticas maiores que 15\% ou, ainda, deslocamentos excessivos. $\mathrm{O}$ valor do incremento pode também ser aumentado, caso o processo venha a convergir de forma sistemática na primeira iteração. O controle dos passos de carga foi efetuado a partir da opção " $T i$ me increment", onde foram admitidos os seguintes valores: Time Step Size = 1,0; Minimum Time Step =0,1 e Maximum Step Size = 1,0. Utilizou-se como parâmetro de convergência uma tolerância igual a 0,001.

\section{RESULTADOS E DISCUSSÃO}

\subsection{Programa experimental}

O comportamento das curvas força versus deslocamento obtidos, para os corpos de prova ensaiados ao cisalhamento direto até a ruptura, com dois pares de conectores X, com $11 \mathrm{~mm}$ de diâmetro, são apresentados na Figura 12.

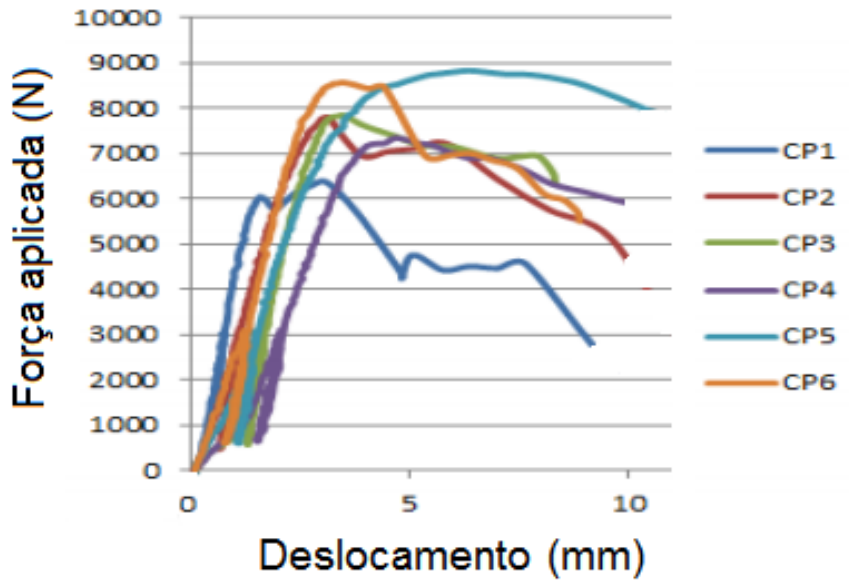

Figura 12: Curvas força versus deslocamento para os corpos de prova de Eucalipto urograndis com dois conectores em $X$ formados por parafusos de $11 \mathrm{~mm}$ de diâmetro (marca Rothoblass). Fonte: CALIL NETO [5].

A Tabela 3 apresenta os resultados experimentais das forças que provocam uma deformação de $2 \%$ nos corpos de prova (CP 1 a CP6) ensaiados experimentalmente ao cisalhamento direto (Direct shear), segundo recomendações da ABNT NBR 7190 [7]. Os resultados, neste caso, são apresentados para dois pares de conectores $\mathrm{X}$.

Tabela 3: Força de ruptura dos corpos de prova referentes a deformação 2\%o segundo ABNT NBR 7190 [7].

\begin{tabular}{l|l}
\hline CORPO DE PROVA & $\begin{array}{l}\text { VALOR DAFORÇA } \\
(\mathrm{kN})\end{array}$ \\
\hline $\mathrm{CP} 1$ & 78 \\
\hline $\mathrm{CP} 2$ & 46 \\
\hline $\mathrm{CP} 3$ & 90 \\
\hline $\mathrm{CP} 4$ & 93 \\
\hline $\mathrm{CP} 5$ & 95 \\
\hline CP6 & 81 \\
\hline \multicolumn{2}{l}{ Sendo: $\mathrm{kN}$ (Kilonewton) }
\end{tabular}

Na Tabela 4 apresentam-se as médias, os coeficientes de variação (CV) e os valores mínimos (MIN) e máximos (MAX) obtidos para o tratamento T7 (Tabela 1).

Tabela 4: Resultados da análise estatística obtida para a força de ruptura dos corpos de prova. Fonte CALIL NETO [5]. 


\begin{tabular}{l|l|l|l}
\hline $\begin{array}{l}\text { VALOR MÉDIO } \\
(\mathrm{kN})\end{array}$ & CV & MIN & MAX \\
\hline 80,05 & 15 & 68,67 & 90,08 \\
\hline \multicolumn{2}{l|l}{ Sendo: kN (Kilonewton) } &
\end{tabular}

Sendo: kN (Kilonewton)

Por meio da análise estatística, realizada nos ensaios, foi possível constatar que a espécie de madeira e a orientação da fibra são fatores que causam variação, significativa, na resistência da ligação. Os resultados experimentais mostraram, também, que o ângulo de $45^{\circ}$, entre a força aplicada e a posição de penetração do parafuso, conduz a melhores resultados de resistência dos parafusos, visto que os conectores são solicitados à tração e à compressão, neste caso.

\subsection{Programa numérico}

A calibração do modelo numérico foi feita a partir da comparação numérico-experimental da curva Força versus Deslizamento, obtida entre as peças lateral e central do corpo de prova. Para tanto, a curva "numérica", para o modelo analisado, foi plotada até o ponto máximo, onde foi possível a obtenção de convergência dos resultados em correspondência a uma tolerância admitida de 0,001 . A Figura 13 apresenta a comparação das curvas, numérica e experimental média, obtidas para corpos de prova com dois conectores em X.

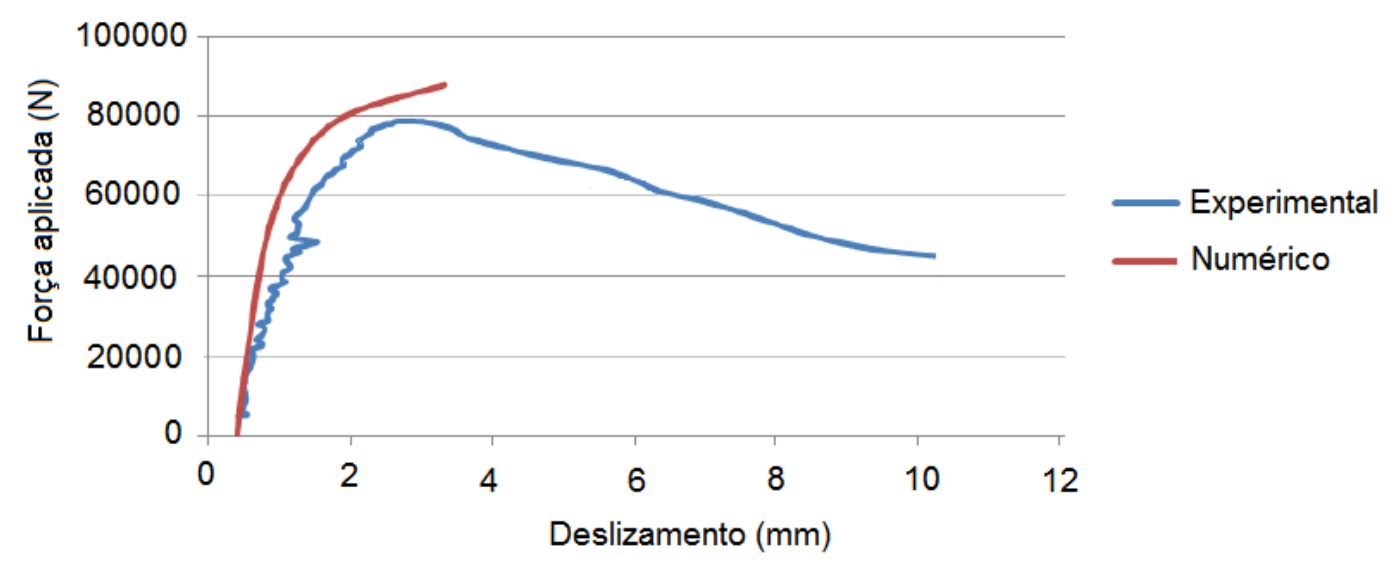

Figura 13: Comportamento da curva numérica força versus deslizamento para corpo de prova com 2 conectores em X.

A relação entre Força e Deslocamento, obtida para o modelo analisado, apresentou, basicamente, uma fase inicial com comportamento linear e, a partir de uma determinada força, a relação começou a apresentar um comportamento não linear, devido a não linearidade física dos materiais. No entanto, observou-se, para a curva numérica Força versus Deslizamento, da Figura 13, uma inclinação ascendente no início do trecho não linear, sem apresentação do trecho descendente. Neste caso, por mais que se refine a malha dos elementos componentes do modelo numérico, não se obtém melhoras no comportamento da curva. Isso se deve ao fato do elemento finito solid45, considerado na modelagem das peças de madeira e dos conectores de cisalhamento, ser um elemento simples e pouco flexível. Além disso, foram utilizados valores aproximados para as propriedades plásticas dos materiais, sendo estes valores, no caso da madeira, determinados com base na resistência da madeira na compressão. Por outro lado, a simulação numérica do comportamento da madeira não é uma tarefa simples, devido à possibilidade de existência de imperfeições (distorções nas direções das fibras e presença de nós) que influenciam no seu comportamento. A madeira é caracterizada por três direções ortogonais (longitudinal, tangencial e radial) tendo, cada uma das direções do material, propriedades mecânicas diferentes das outras duas direções, como, também, comportamentos diferentes na tração e na compressão. $\mathrm{Na}$ compressão, o comportamento da madeira é relativamente plástico, podendo ser aproximado por uma lei constitutiva elasto-plástica com endurecimento. Na tração, o comportamento da madeira é frágil e, neste caso, o modelo elasto-plástico não representa, verdadeiramente, o comportamento do material.

Os valores de rigidez $\mathrm{k}$ (força versus deslocamento) de dois conectores $\mathrm{X}$, obtidos a partir das curvas experimental e numérica da Figura 13, para carregamentos de serviço (trecho linear) foram, respectivamente, iguais a $25,86 \mathrm{KN} / \mathrm{mm}$ e $35,32 \mathrm{KN} / \mathrm{mm}$. Já os valores experimental e numérico da força de ruptura, referentes à deformação de $2 \%$ para dois conectores $\mathrm{X}$, foram iguais a $79,86 \mathrm{kN}$ e $80,05 \mathrm{kN}$, respectivamente. No entanto, as máximas forças atingidas pelos corpos de prova, de acordo com a Figura 13, foram de, aproxima- 
damente, $80 \mathrm{kN}$ e $88 \mathrm{kN}$, respectivamente. A avaliação das tensões no modelo efetuada para todo o corpo de prova e, principalmente, para as regiões dos conectores de cisalhamento é apresentada na Figura 14.
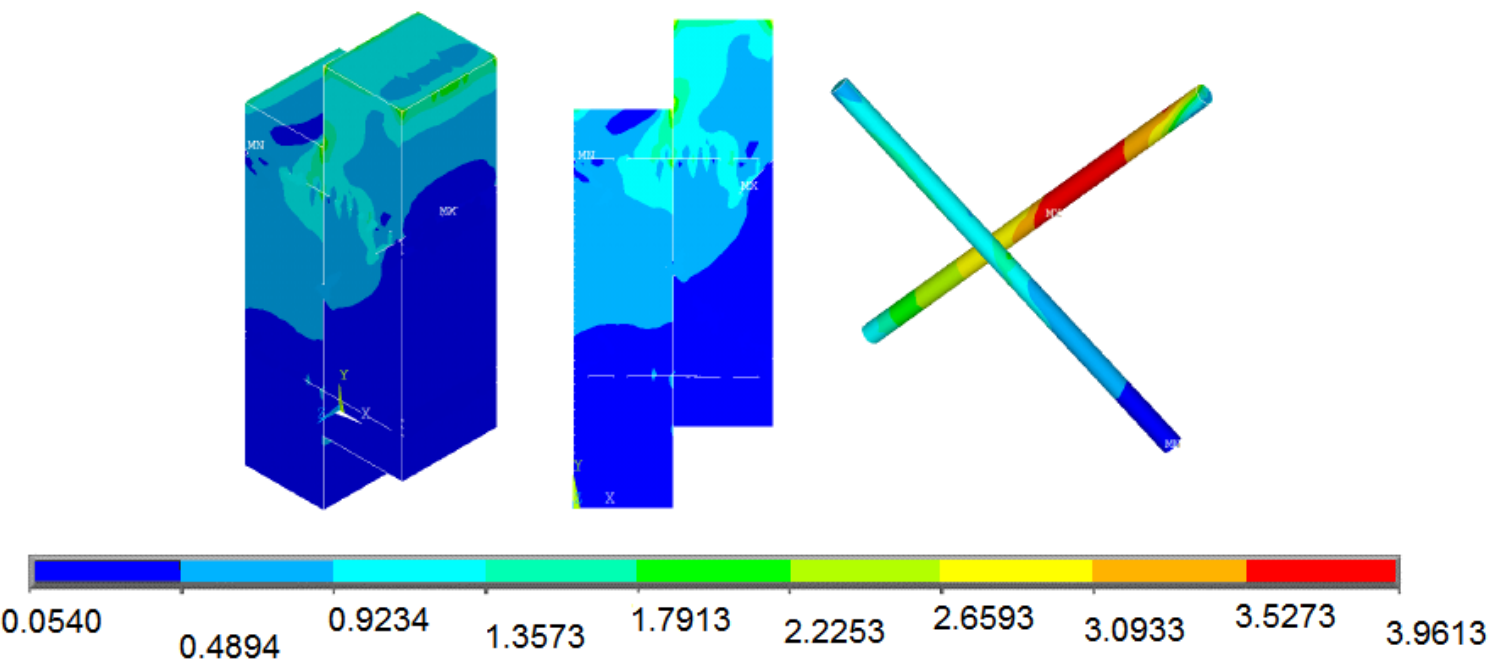

Figura 14: Distribuição das tensões $\left(\mathrm{kN} / \mathrm{cm}^{2}\right)$ obtidas numericamente no ANSYS [4] para o corpo de prova com conectores dispostos em $\mathrm{X}$.

A partir da Figura 14, para o carregamento aplicado na análise numérica do corpo de prova, pode-se observar que, no conector em " $x$ ", o parafuso comprimido tendeu a apresentar uma rótula plástica (envergadura em função da tensão aplicada na interface da madeira), enquanto que, no parafuso tracionado, isso não ocorreu, pois o conector tendeu a ser arrancado. O maior valor de tensão $\left(3,9613 \mathrm{kN} / \mathrm{cm}^{2}\right)$ foi observado no parafuso comprimido. Neste caso, para o nível de carregamento aplicado ao corpo de prova em análise, a superfície superior do mesmo esteve sujeita a níveis de tensão mais baixos $\left(0,9234 \mathrm{kN} / \mathrm{cm}^{2}\right)$, indicando, assim, que mesmo quando todo o corpo de prova como um todo trabalha dentro do regime linear, quando da aplicação de um determinado carregamento, a região dos conectores atinge o limite plástico do material. Resultados numéricos semelhantes para os conectores de cisalhamento foram obtidos por MOLINA [1] e MIOTTO [13].

\section{CONCLUSÕES}

O modelo numérico proposto neste trabalho permitiu a avaliação do comportamento do sistema de conexão em X, formado por dois parafusos auto-atarraxantes de $11 \mathrm{~mm}$, inclinados a $45^{\circ}$, em corpos de prova de cisalhamento confeccionados com madeira laminada colada de Eucalipto urograndis.

O modelo permitiu a análise do sistema de conexão em termos de resistência como, também, de rigidez $\mathrm{k}$ (força versus deslizamento). O modelo permitiu, principalmente, a avaliação dos níveis de tensão nas regiões dos conectores, complementando, assim, a análise experimental. O modelo numérico em questão permitiu, também, a obtenção dos valores internos de tensão em outras regiões do corpo de prova, os quais não são obtidos facilmente em análises experimentais.

A partir do modelo numérico proposto, não foi possível a obtenção do comportamento descendente da curva Força versus Deslizamento, para valores aplicados após a força de ruptura, pois o modelo considera apenas as propriedades elásticas e plásticas do material, não considerando as propriedades relativas aos danos e mecânica da fratura. Esse comportamento se deve, basicamente, ao fato do elemento finito solid45, utilizado na modelagem das peças de madeira e aço, ser um elemento relativamente simples.

Os valores numéricos da resistência convencionada a deformação $2 \%$, obtidos para dois conectores X, foram muito próximos dos valores experimentais, onde as diferenças entre tais valores foram da ordem de $1 \%$. Os valores numéricos e experimentais de rigidez $\mathrm{k}$ (força versus deslizamento), para dois conectores $\mathrm{X}$, apresentaram diferenças de, aproximadamente, $27 \%$, sendo os valores numéricos superiores aos valores experimentais. 


\section{AGRADECIMENTOS}

Os autores agradecem a Fundação para o Incremento da Pesquisa e do Aperfeiçoamento Industrial (FIPAI) pelo financiamento da pesquisa.

\section{BIBLIOGRAFIA}

[1] MOLINA, J.C., Análise do comportamento dinâmico da ligação formada por barras de aço coladas para tabuleiros mistos de madeira e concreto para pontes, Tese de D.Sc., EESC/USP, São Carlos, SP, Brasil, 2008.

[2] FRANGI, A., KNOBlOCH, M., FONTANA, M., "Fire Design of Timber-Concrete Composite Slabs with Screwed Connections", Journal of structural engineering, v. 136, n. 2, pp. 219-228, Feb. 2010.

[3] TOMAS, U., HANS, J.B., "Determining suitable spacings and distances for self-tapping screws by experimental and numerical studies", In: Proceedings of the World Conference on Timber Engineering, pp. 1-11, Riva del Guarda, Jun. 2010.

[4] ANSYS INC. “Ansys Release 10.0”. Documentation. 2005.

[5] CALIL NETO, C., Ligações com parafusos auto-atarraxantes se pré-furação para uso em estruturas de madeira, Tese de D.Sc., EESC/USP, São Carlos, SP, Brasil, 2014.

[6] ROTHOBLASS. Couso fixing safe house. In: Couso Projettazione Connecione, Cortaccia, 2012.

[7] ASSOCIAÇÃO BRASILEIRA DE NORMAS TÉCNICAS. Projeto de Estruturas de Madeira: NBR 7190. Rio de Janeiro, 1997.

[8] TRUEGRID versão 2.10 Documentation. 2001.

[9] TGEdit, “True Grid Edit Mesh - File, versão 2.2, Software Free. 2001.

[10] BALARIN, A.W., NOGUEIRA, M., "Caracterização elástica da Madeira de Eucalipto citriodora”, Cerne, v. 9, n. 1, pp. 066-080. 2003.

[11] DIAS, A. M. P. G., Mechanical behavior of timber-concrete joints, Tese D.Sc., University of Coimbra Coimbra, Coimbra, Portugal, 2005.

[12] FLORES, E. S., RIOSECO, C. A., MATAMAL, A. V., "Calibración del modelo de Hill modificado para el Pino Radiata cileno en conexiones de cizalle doble sometidas a compressión paralela”, In: II Jornadas Chilenas de Estructuras de Madera, pp. 1-10, Santiago, Nov. 2007.

[13] MIOTTO, J.L., Estruturas mistas de madeira-concreto: Avaliação das vigas de madeira laminada colada reforçadas com fibra de vidro, Tese de D.Sc., EESC/USP, São Carlos, SP, Brasil, 2009. 